\title{
EMBEDDING ENVIRONMENTAL ACTIONS IN TIME AND SPACE: THE EVOLUTION OF SUSTAINABILITY NARRATIVES
}

\author{
DÉBORAH PHILIPPE \\ University of Lausanne \\ Faculty of Business and Economics \\ 1015 Lausanne, Switzerland \\ PRATIMA BANSAL \\ University of Western Ontario

\section{INTRODUCTION}

The question of how organizations manage the complexity of their relationships with the natural environment has become one of the most topical issues of recent research on management, as evidenced by the number of papers, special issues or books that investigate the nature and extent of interactions between organizations and the natural environment. However, the ontological underpinnings of the organization-environment relationships remain surprisingly under-discussed and we are still missing a microlevel account of the processes by which actors identify and make sense of these relationships across time and space (Whiteman \& Cooper, 2011).

In spite of the different ways in which the integration of business and the environment can be configured, most work assume a weak form of sustainability, which "sets out to bring environmental concerns into the framework provided by the structures and systems of business" (Roome, 2012: 620-621). This approach constitutes an expansion of the dominant and traditional vision of environmental and social worlds as relatively distinct from the organizational one and pays little attention to the larger spatiotemporal context in which corporate environmental actions are embedded. In contrast, the strong form of sustainability advocated by scholars acknowledging the interdependence between organizational, environmental, and social systems (e.g., Gladwin et al., 1995; Purser, Park, and Montuori, 1995; Shrivastava, 1995; Starik and Rands, 1995), "seeks to integrate the company into environmental or socio-ecological systems" (Roome, 2012: 620-621). Whereas weak sustainability tends to focus on local, short-term outcomes, strong sustainability can accommodate the larger spatiotemporal context in which corporate environmental actions are embedded, which has strong implications for the nature and sustainability of organizational activities.

Because the past two decades of work on corporate environmentalism has largely overlooked the distinctiveness of these two paradigms (Roome, 2012) and has rather embraced a weak sustainability paradigm, we are left with no cogent understanding of how organizations handle the increasing complexity of their interactions with the natural environment. Specifically, since the research community has mainly approached sustainability in its weak form, there is little research that attempts to unpack the empirical differences between these two paradigmatic perspectives and similarly, little research that investigates which form of sustainability organizations embrace.

The present study attempts to address these gaps by drawing on a longitudinal, inductive analysis of the environmental discourses of 9 Canadian organizations from 1986 to 2010 and investigating how representations of organization-environment interactions are built into 
organizational narratives. The concept of organizational narrative and the idea of understanding organizational life as "interwoven, structured, and even construed by stories and narratives" have largely entered organizational studies (Hartz and Steger, 2010: 768) and discourse-based methods have become increasingly popular when investigating organizational phenomena (e.g., Barley, 1983; Brown, Ainsworth, and Grant, 2012; Kilduff, 1993; Heracleous and Barrett, 2001; Phillips and Brown, 1993; Shipp and Jansen, 2011; Suddaby and Greenwood, 2005; Vaara, 2002). In this study, we use a structural approach to organizational narratives, drawing from Greimas' works on narrative semiotics and structural semantics (Greimas, 1971). This particular approach to narratives provides deep insights into the organizations' sensemaking processes.

\section{THEORY}

Research that has addressed the question of how organizations manage their relationships with the natural environment has tended to follow one of three approaches, which can be classified as antecedent-focused, outcome-focused, and ontology-focused.

The antecedent-focused approach investigates the antecedents of the organizations' environmental actions. Research within this field emphasize both the role of managerial values, beliefs, and interpretations (e.g., Bansal and Roth, 2000; Cordano and Frieze, 2000; Egri and Herman, 2000; Flannery and May, 2000; Sharma and Henriques, 2004) and the role of contextual elements (e.g., Bansal and Roth, 2000; Hoffman, 1999; Sharma, 2000) in shaping organizations' responses to environmental issues.

The outcome-focused approach to corporate environmentalism focuses on the impacts of corporate environmentalism on several organizational outcomes, such as financial performance (e.g., Judge and Douglas, 1998; Russo and Fouts, 1997), competitive advantage (e.g., Christmann, 2000), or social evaluations (Philippe and Durand, 2011). Similarly, a significant body of research examines the impacts of corporate environmentalism on environmental outcomes such as toxic releases, waste management, or the adoption of ISO 14001 (Christmann and Taylor, 2001; King and Lenox, 2000; King and Shaver, 2001).

The third and more limited ontology-focused approach analyses changes in the mindsets required to succeed in building ecologically sustainable organizations (Gladwin et al., 1995; Purser, Park, and Montuori, 1995; Shrivastava, 1995; Starik and Rands, 1995). Scholars in this stream of research have developed a multilevel, multisystemic understanding of the relationships between organizations and the natural environment. They argue that the relationships between society and the environment are complex, and economic activities require integrative, systems thinking to achieve sustainability at all levels of analysis.

Although studies pertaining to the first two approaches offer valuable foundations for appreciating the antecedents and consequences of eco-responsible actions, they largely ignore the larger context in which these actions are embedded. The causal models they develop tend to favor an atomistic view of the world and of organizations' actions and as such are mainly anchored in a weak sustainability paradigm.

This lack of attention vested on the context in which corporate environmental actions are embedded raises the question of the paradigm that organizations embrace when handling their relationships with the natural environment. Specifically, do they operate in a weak sustainability mindset, as could be deduced from the vast majority of empirical studies that look at these actions in an atomistic fashion? Or, do they operate in a stronger sustainability mindset, that 
recognizes the inherent complexity of these interactions and therefore handle them in a more systemic and integrative approach?

To answer this question, it is important to understand how organizations make sense of their interactions with the natural environment. Sensemaking is a critical organizational activity (Weick, 1995) which refers to a process of social construction (Berger \& Luckmann, 1967) in which actors engage in the interpretation and explanation of cues from their environment in an effort to create order and retrospectively understand past events (Weick, 1993). Organizational sensemaking is fundamentally a social and linguistic process (Chia, 2000; Maitlis, 2005), where meanings are materialized through language, talk, and communication (Weick, Sutcliffe \& Obstfeld, 2005). As organizations plan and enact narratives that are consistent with their values and beliefs (Czarniawska, 1997), these narratives also provide a window onto these organizations' worldviews or paradigms (Gioia \& Chittipeddi, 1991; Pentland, 1999), that is "the constellation of beliefs, values and concepts that give shape and meaning to the world [an actor] experiences and acts within" (Norton, 1991: 75). As evidenced by Morgan (1980) and Schon and Rein (1994), these worldviews "rarely take the form of highly developed systematic philosophies" but typically remain "sets of background assumptions that tend to organize language, thoughts, perceptions, and actions" (Gladwin et al., 1995: 880). Investigating how organizations structure their discourses on corporate environmentalism can therefore provide us with insights into the sustainability paradigm they embrace.

\section{METHODS}

In this study, we examine the environmental communication of a set of Canadian organizations belonging to the resource industry. The sample comprises 9 organizations operating in three different sectors: 1) forestry, paper, and forest products; 2) oil and gas; and 3) mining. The main source of data consists in the annual reports of these 9 organizations from 1986 to 2010. Following Tolbert and Zucker (1983) and Bansal (2005), an analysis of the annual reports was carried out for 9 years - 1986, 1989, 1992, 1995, 1998, 2001, 2004, 2007 and 2010. The earliest year, 1986, was chosen because awareness of sustainable development was very limited prior to 1987 - the year that sustainable development was popularized by the publication of the Brundtland Commission report and by the Montreal Protocol. The final year, 2010, is the last year for which organizations released an annual report during the data collection period. The collect yielded 80 reports (due to a merger in 2009, one of the organizations did not publish its report in 2010).

We carried out the data analysis in two main stages. In the first stage, we identified through a traditional content analysis, actions involving organization-environment interactions in each annual report. The first author and two independent trained coders identified and listed all organizations' actions implying any connection to the natural environment. We selected actions as our unit of analysis in order to unveil a comprehensive and fine-grained picture of how organizations make sense of corporate environmentalism by discursively forging connections between otherwise separate events. We identified a total of 1198 actions carried on by the focal 9 organizations across our period of observation.

The second stage of the data analysis had two parts. The first part involved defining the actantial dimension of each action while the second part involved identifying the spatiotemporal context in which this action was embedded. In order to do this, we performed a structural analysis of the organizational narratives contained in the annual reports. Structural semantics 
hold that exploring the narrative structure of a textual corpus allows for the apprehension of the meaning of a text and its underlying values. To fully capture the meaning of a narrative, it is not sufficient to rely solely on traditional content-based analyses. Instead, it is necessary to also take into account the different elements that constitute its structure. The structuralist perspective provides such an insight into the formal organization of a narrative by conceptualizing narratives as a large sentence that can be formally and grammatically analysed as such. That is, given the remarkable recurrence of specific narrative characteristics, the narrative can be studied as having a structure of development similar to the syntactic structure of a sentence (Greimas, 1971). This narrative grammar, which is one of the fundamental principles of the internal organization of discourses, addresses the Aristotelian trinomial nexus of drama: action, time, and space.

Action is the fundamental dimension of a narrative: who performs the action and how the action is performed are central elements of the storytelling process. To study this agentic dimension, Greimas (1966) proposes a theoretical model that can be applied to all types of narratives. His actantial model postulates different modalities of action, which refer to propositions that express different modal categories of "doing" thus governing and specifying on a syntactic level the relationship between the focal actant (in our context, the organization) and the action it performs. We distinguished between four semantic investments of "doing": volitionto-do and its opposite, obligation-to-do, both referring to the wanting modality; knowledge-to-do, referring to the knowing modality; and power-to-do, referring to the being able modality. Attributions of agency were realized grammatically through verbal, adverbial and any other semantic constructions reflecting the different modalities. The obligation-to-do modality was used to characterize actions mandated or instigated by external forces (e.g., social pressure, regulations, market demand) while the volition-to-do modality was used to describe actions instigated by the focal organization. Actions for which it was indicated that the focal organization either had the power to perform it or had gained power through it, was coded as expressing a power-to-do modality. Eventually, the knowledge-to-do modality was attributed to actions for which it was indicated that the focal organization either had the knowledge to perform the action or had gained knowledge through it.

In order to grasp the underlying meaning of a narrative, it is crucial to understand how discrete actions are related to other actions or events. Doing so requires looking at the spatiotemporal context of actions. Together with the agentic dimension, the discursive syntax of a narrative comprises processes of temporalization and spatialization. That is, actions are articulated through time and space in order to produce meaning. We generalize Greimas' description by considering the narrative as a sequence of actions which are temporally and spatially situated. The second part of our structural analysis thus consisted in mapping environmental actions into temporal and spatial structures. The objective of such mapping was to unravel the extent of spatiotemporal embeddedness of each environmental action. The temporal mapping consisted in assessing whether a focal action was connected to other actions performed or planned by the organization, that is, whether the action was embedded in the organization's history. For each action, we thus coded for the presence or absence of any mention to another past or future action by the organization. The spatial mapping consisted in assessing whether a focal action was connected to actions performed by other actors within the field (i.e., stakeholders), that is, whether the action was embedded in the larger organizational field. For each action, we thus recorded whether it had been performed by the focal organization on its own or whether collaboration with other actors was mentioned. 


\section{DISCUSSION OF FINDINGS}

Across the period, we observed the emergence and development of sustainability stories. We found relatively few temporal and spatial connections at the beginning (approximately from 1986 to the mid-90s) but in the later years, corporate environmentalism is framed as an enduring component of the organization's history and as a part of a larger system in which other actors operate. The sensemaking processes in which organizations are engaged throughout the period appear richer and are characterized by greater levels of discursive integration, leading to the formation of polyphonic, yet coherent and unified sustainability narratives. The different narrative structures we uncovered constitute frames within which organizations make sense of their relationships with the natural environment.

The way organizations forge temporal and spatial connections between actions is representative of their worldviews, that is, of the paradigm they embrace. Compared to weak sustainability, strong sustainability is less amenable to the control of a single organization but instead requires "new ways to appreciate the complex relationships and connections between the parts of a system and the function of the whole" (Ackoff, 1999, in Roome, 2012: 625) built on a form of organizational learning that involves collaboration between multiple actors (Roome, 2012). We add to this definition that strong sustainability also requires organizations to appreciate the connections between past, present, and future. Our structural analysis led us to identify the time-space interfaces of these two paradigms. If the weak sustainability paradigm can be characterized by spatiotemporal discontinuities, the strong sustainability paradigm is embodied by spatiotemporal connections. Our finding that organizations increasingly forge stories in which they highlight temporal connections between their different environmental actions and engage in collaboration with different stakeholders therefore indicates that organizations are increasingly embracing a strong sustainability approach to frame their interactions with the natural environment.

Another important finding of our study concerns the nature of corporate environmental agency and specifically the use of modalities of action. If the volition-to-do and obligation-to-do modalities were the most frequently used over the period to refer to corporate environmental actions, the use of the power-to-do and knowledge-to-do modalities significantly increases (they represented $20 \%$ of the modalities in 1986 and $42 \%$ in 2010). Interestingly, we found that increases in the use of these two modalities (which we refer to as strategic capabilities) were associated with higher levels of spatiotemporal embeddedness. Specifically, we find that not only do organizations that embed their environmental actions in time or space also display greater levels of strategic capabilities, but also that these strategic capabilities are almost always used for actions that are temporally or spatially embedded.

A potentially powerful framework for interpreting our findings and discussing their implications is the literature on organizational boundary-spanning (e.g., Aldrich \& Herker, 1977; Dollinger, 1984; Tushman \& Scanlan, 1981). A central argument in this research area is that organizations that are able to span across organizational boundaries (i.e., to draw connections between the organization and its external environment) are better equipped for survival because their higher tolerance for ambiguity (Dollinger, 1984), access to novel sources of information (Tushman \& Scanlan, 1981), and reliance on more sophisticated information processing capabilities and external representation mechanisms (Aldrich \& Herker, 1977) allow them to increase "[their] ability to learn and perform according to changing contingencies in the environment" (Terreberry, 1968: 590). 
This argument is consistent with our finding that spatiotemporal embeddedness is associated with higher levels of strategic capabilities. Organizations that embed their environmental actions in time, by drawing connections between past, present, and future actions, can be conceptualized as engaged in temporal boundary-spanning activities. Similarly, organizations that embed their environmental actions in space, by interacting and collaborating with stakeholders, can be conceptualized as engaged in spatial boundary-spanning activities.

Here, we propose that organizations that engage in spatiotemporal boundary-spanning (i.e., that embrace a strong sustainability paradigm) will be less vulnerable to external changes because they understand the porosity of their temporal and spatial boundaries and as such are able to develop over time a systemic and integrative understanding of their relationships with the natural environment. By embedding their environmental actions in time and space, they can access a richer repertoire of actions, skills, and meanings and are therefore better able to engage with the tensions inherent to sustainability and adapt to changing expectations.

This boundary spanning perspective on corporate environmentalism resonates with Whiteman and Cooper's findings (2011) that actors that are ecologically embedded face better opportunities to increase their repertoire of skills, are more sensible to changes to their environment, better able to anticipate surprises, and therefore better equipped to adapt to changing contextual conditions. This approach also resonates with a recent study by Slawinski and Bansal (2012) that shows how an organization's temporal perspective relates to its responses to climate change issues. Specifically, the authors distinguished between two types of organizations: "focused" organizations that favored a linear time perspective and for which past and future were not salient aspects to present decision-making, and "integrated" organizations that favored a cyclical time perspective and which drew on past events and future scenarios in their decision-making processes. They found that "focused" organizations' responses to climate change were narrower in breadth than those implemented by "integrated" organizations. However, although in both of these studies, time and space jointly play a role in actors' environmental behavior, the intersecting nature of time and space remains under-discussed. In contrast, our findings point to the interconnected nature of time and space in the context of corporate environmentalism.

Together, the findings of our study highlight the potential for the development of theory and research that integrate time and space in investigations on the relationships between organizations and the natural environment. We also point to the potential of the boundaryspanning literature to provide key insights into corporate environmentalism and sustainability research. Eventually, we call for the necessity to develop research that embrace a paradigm more suited to account for the systemic nature of the interactions between organizations and the natural environment.

\section{REFERENCES AVAILABLE FROM THE AUTHOR(S)}

